\title{
A Lactobacillus casei Shirota probiotic drink reduces antibiotic-associated diarrhoea in patients with spinal cord injuries: a randomised controlled trial
}

\author{
Samford Wong ${ }^{1,2,3 *}$, Ali Jamous ${ }^{1}$, Jean O'Driscoll ${ }^{4}$, Ravi Sekhar ${ }^{5}$, Mike Weldon ${ }^{5}$, Chi Y. Yau ${ }^{6}$, \\ Shashivadan P. Hirani ${ }^{2}$, George Grimble ${ }^{3}$ and Alastair Forbes ${ }^{3}$ \\ ${ }^{1}$ National Spinal Injuries Centre, Stoke Mandeville Hospital, Aylesbury HP21 8AL, UK \\ ${ }^{2}$ School of Community and Health Science, City University, London EC1V OHB, UK \\ ${ }^{3}$ Centre for Gastroenterology and Clinical Nutrition, University College London, London WC1E 6BT, UK \\ ${ }^{4}$ Department of Microbiology, Stoke Mandeville Hospital, Aylesbury HP21 8AL, UK \\ ${ }^{5}$ Department of Gastroenterology, Stoke Mandeville Hospital, Aylesbury HP21 8AL, UK \\ ${ }^{6}$ Medicine for Older People, Stoke Mandeville Hospital, Aylesbury HP21 8AL, UK \\ (Submitted 23 April 2013 - Final revision received 5 August 2013 - Accepted 12 August 2013 - First published online 18 September 2013)
}

\section{Abstract}

Certain probiotics may prevent the development of antibiotic-associated diarrhoea (AAD) and Clostridium difficile-associated diarrhoea (CDAD), but their effectiveness depends on both strain and dose. There are few data on nutritional interventions to control AAD/CDAD in the spinal cord injury (SCI) population. The present study aimed to assess (1) the efficacy of consuming a commercially produced probiotic containing at least $6.5 \times 10^{9}$ live Lactobacillus casei Shirota (LCS) in reducing the incidence of AAD/CDAD, and (2) whether undernutrition and proton pump inhibitors (PPI) are risk factors for AAD/CDAD. A total of 164 SCI patients (50.1 (SD 17.8) years) with a requirement for antibiotics (median $21 \mathrm{~d}$, range 5-366) were randomly allocated to receive LCS ( $n$ 76) or no probiotic ( $n$ 82). LcS was given once daily for the duration of the antibiotic course and continued for 7 days thereafter. Nutritional risk was assessed by the Spinal Nutrition Screening Tool. The LcS group had a significantly lower incidence of AAD (17.1 $v .54 \cdot 9 \%, P<0 \cdot 001)$. At baseline, $65 \%$ of patients were at undernutrition risk. Undernutrition $(64 \cdot 1 v .33 \cdot 3 \%, P<0 \cdot 01)$ and the use of PPI $(38 \cdot 4 v \cdot 12 \cdot 1 \%, P=0 \cdot 022)$ were found to be associated with AAD. However, no significant difference was observed in nutrient intake between the groups. The multivariate logistic regression analysis identified poor appetite $(<1 / 2$ meals eaten) (OR 5.04, 95\% CI 1.28, 19.84) and no probiotic (OR 8.46, 95\% CI $3 \cdot 22,22 \cdot 20)$ as the independent risk factors for AAD. The present study indicated that LcS could reduce the incidence of AAD in hospitalised SCI patients. A randomised, placebo-controlled study is needed to confirm this apparent therapeutic success in order to translate into improved clinical outcomes.

Key words: Probiotics: Spinal cord injuries: Diarrhoea: Undernutrition risk

Antibiotic-associated diarrhoea (AAD) is a common complication of antibiotic use ${ }^{(1-5)}$. The frequency of AAD can be as high as $60 \%$ during hospital outbreaks or intermediate (13-29\%) during endemic periods ${ }^{(1)}$.

Risk factors for AAD include the use of broad-spectrum antibiotics, various host factors (e.g. old age), a longer hospitalisation period and exposure to nosocomial pathogens ${ }^{(2)}$. Diarrhoea associated with antibiotic use and caused by Clostridium difficile is also a complication of treatment with antimicrobial agents and has been reported in up to $25 \%$ of patients $^{(3)}$. Once this ecosystem of micro-organisms is disturbed, patients become more susceptible to infection with opportunistic pathogens.

C. difficile is a spore-forming, Gram-positive anaerobe that is a major cause of $\mathrm{AAD}$, and specifically of $C$. difficileassociated diarrhoea (CDAD) in hospitals. It is a particularly virulent pathogen because it produces an enterotoxin and a cytotoxin, both of which cause mucosal injury and damage to the colon. When antibiotic therapy disrupts this natural defence, $C$. difficile multiplies and produces toxins, causing diarrhoea. In some cases, severe inflammation ( $C$. difficileassociated colitis) can develop ${ }^{(6)}$.

Abbreviations: AAD, antibiotic-associated diarrhoea; CDAD, Clostridium difficile-associated diarrhoea; CFU, colony-forming units; LcS, Lactobacillus casei Shirota; PPI, proton pump inhibitor; SCI, spinal cord injury; SNST, Spinal Nutrition Screening Tool.

*Corresponding author: S. Wong, fax +44 1296 315049, email samford.wong@ucl.ac.uk 
There is growing interest in probiotics to reduce the risk of $\mathrm{AAD}$ and CDAD, because of their wide availability as safe dietary supplements, and concern over recent outbreaks of severe $C$. difficile in the $\mathrm{UK}^{(7)}$. Probiotics are defined as live microorganisms which, when administered in adequate amounts, confer a health benefit on the host ${ }^{(8)}$. Probiotics are thought to be beneficial via mechanisms linked to the recolonisation of the gut mucosa, the restoration of the gut microbiota equilibrium, the competitive exclusion of pathogens and toxins from the host and the re-establishment of the luminal metabolome ${ }^{(4,9-12)}$.

Early studies for the prevention and treatment of AAD, which identified the use of proton pump inhibitors (PPI) ${ }^{(12)}$ and undernutrition $^{(13)}$ as the possible risk factors for AAD/CDAD, have failed to provide a clear result. A more recent metaanalysis that included eighty-two randomised controlled trials was therefore performed ${ }^{(14)}$. Of the eighty-two randomised controlled trials, seventeen involved Lactobacillus-based interventions. The meta-analysis found that adjunctive probiotic administration was associated with a reduced risk of AAD (relative risk 0.64, 95\% CI 0.47, 0.86), but that there was a variation in the trial design, type of probiotic strains, dosage, duration of treatment and study population, presumably explaining much of the prior uncertainty ${ }^{(12)}$.

To the best of our knowledge, data on the prevalence of AAD in spinal cord injury (SCI) patients are limited, and no studies have examined the effect of probiotics in preventing diarrhoea in SCI patients ${ }^{(15)}$. It appeared logical to assess probiotics in SCI patients because these patients are particularly vulnerable to diarrhoea and its consequences for many reasons ${ }^{(16)}$. Diarrhoea can delay rehabilitation, increase the risk of developing pressure ulcers/delay wound healing and reduce quality of life ${ }^{(16)}$.

We therefore planned a randomised controlled trial to assess the efficacy of a commercial probiotic (LcS, Lactobacillus casei Shirota) for the prevention of AAD and CDAD in adult patients with SCI. The objectives of the present study were to determine (1) the potential efficacy of LcS for the prevention of $\mathrm{AAD}$ and CDAD for the duration of antibiotic use, and for $30 \mathrm{~d}$, and (2) whether undernutrition risk and the use of PPI are the risk factors for $\mathrm{AAD}$ and $\mathrm{CDAD}$.

\section{Materials and methods}

Adult patients who sustained a SCI and who were admitted to the National Spinal Injuries Centre at Stoke Mandeville Hospital were screened. The inclusion criteria were in-patients aged $>18$ years old who had sustained a SCI less than 6 months before and who were due to receive antibiotics for infection.

Patients were excluded from the study for the following reasons: (1) diarrhoea before antibiotic therapy; (2) antibiotics for prophylaxis; (3) bowel pathology that could result in diarrhoea; (4) bowel surgery in the last 6 months; (5) infective endocarditis; (6) regular probiotic use in the previous 8 weeks; (7) pancreatitis; (8) active inflammatory bowel disease; (9) immunosuppression; (10) nil by mouth/non-functioning gut; (10) known cows' milk protein intolerance; (11) those who were unable to give informed consent due to cognitive impairment; (12) those with acute stroke.

\section{Study design}

Between September 2010 and September 2012, after obtaining written informed consent, a total of 164 SCI patients, who were within $24 \mathrm{~h}$ of commencing antibiotics, were randomly allocated to receive a probiotic drink (Yakult Light ${ }^{\circledR}: 65 \mathrm{ml}$ ) containing a minimum of $6.5 \times 10^{9}$ colony-forming units (CFU) LcS/ bottle and skimmed milk, or no probiotic (routine care) for the duration of the antibiotic course. When the antibiotic course was finished, a further $7 \mathrm{~d}$ of study drink was prescribed for patients in the active arm of the study. Bowel movements were monitored routinely by the nursing staff on the ward using the Bristol stool chart ${ }^{(17)}$. Where there was evidence of diarrhoea (defined as more than two liquid stools (Bristol Stool Chart type 5, 6 or 7) a day for 3 or more days, in quantities in excess of normal), a stool sample was collected and sent to the microbiology laboratory for the detection of $C$. difficile toxin and glutamate dehydrogenase analysis. Other than in the administration of the yogurt drink, there were no differences in patient care between the two groups.

The principal investigator recorded the occurrence of diarrhoea throughout the study, as documented by the nurses. The census date was fixed $30 \mathrm{~d}$ after the antibiotic course had finished.

The participants' baseline clinical, nutritional and biochemical information were collected. These included age, sex, level of SCI, completeness of injury, American Spinal Injury Association Impairment Scale score, reason for SCI, weight and height, and routine blood tests for total protein and albumin concentrations, $\mathrm{Hb}$, leucocyte count, $\mathrm{Mg}$ and C-reactive protein. Information about nutritional factors, such as route of nutrition, nutrient intake as estimated by food record charts (nil by mouth, less than half, half, more than half and all eaten) ${ }^{(18)}$, interruptions and supplementation of nutrition (use of oral nutritional supplements and artificial nutrition support), was collected. Additional clinical data were recorded, which included the presence of co-morbidities, the use of mechanical ventilation, the history of intensive care unit stay, the number of medications, the name and route of antibiotics and the use of PPI and laxatives.

The intensity of antibiotic exposure was used to categorise patients into those on relatively low-risk antibiotics (metronidazole and parenteral aminoglycosides), those at 'medium-risk' antibiotics (tetracyclines, sulphonamides, macrolides and quinolones) and those at 'high-risk' antibiotics (aminopenicillin and cephalosporins), using the criteria described elsewhere ${ }^{(19)}$.

\section{Definition of undernutrition risk}

Patients were considered at risk of undernutrition on the basis of the Spinal Nutrition Screening Tool (SNST) ${ }^{(20)}$. The SNST assesses eight criteria, of which the majority were recognised as predictors or symptoms of undernutrition: history of recent weight loss; BMI; age; level of SCI; presence of co-morbidity; skin condition; appetite; ability to eat. Each step of screening has a score of up to 5 and the total score reflects the patient's degree of risk. A score of 0-10 indicates a low risk of undernutrition, 11-15 indicates a moderate risk of undernutrition and 
$>15$ indicates a high risk of undernutrition. Patients who had a SNST score $\leq 10$ were considered at low risk and those with a SNST score $\geq 11$ were considered at risk.

\section{Sample size}

Based on the results of a similar UK trial of probiotics (effectiveness of another commercially produced probiotic preparation containing L. casei DN-114001 (L. casei Imunitass; $1.0 \times 10^{8}$ $\mathrm{CFU} / \mathrm{ml})$, Streptococcus thermophilus $\left(1.0 \times 10^{8} \mathrm{CFU} / \mathrm{ml}\right)$ and Lactobacillus bulgaricus $\left(1.0 \times 10^{7} \mathrm{CFU} / \mathrm{ml}\right)$ in the prevention of AAD in general hospitalised patients in November 2002 to January 2005) ${ }^{(21)}$, we estimated that a sample size of 164 was needed, such that a $20 \%$ difference in the proportion of patients developing AAD (30\% in the control group and 10\% with LcS) would be detected with a statistical power of $90 \%$ and at a significance level of $5 \%$.

\section{Statistical analysis}

Descriptive statistics were used to describe the basic features according to age, sex and disease symptoms. $\chi^{2}$ tests were used to compare differences in the distribution of qualitative variables. Differences in quantitative variables, according to their distribution, were analysed by the parametric $t$ test or the non-parametric Mann-Whitney test. Multiple binary logistic regression analysis was used to determine significant predictors for $\mathrm{AAD}$, and effect estimates were presented as the OR and $95 \%$ CI. For all tests, a $P$ value of 0.05 or less or when the $95 \%$ CI for OR did not exceed 1.0 was considered as significant. Statistical analysis was performed using the Minitab statistical software (version 15.0; Minitab, Inc.) and SPSS (version 19; IBM Corporation). All analyses were performed on an intention-to-treat basis

Approximately $10 \%$ of the routine data were lost in the present study (predominantly simple biochemical and haematological variables). To reduce the bias implicit in utilising only complete cases, multiple imputation procedures for the laboratory data were implemented using SPSS (version 19; IBM Corporation) Markov Chain Monte Carlo multiple imputation function to produce five imputed datasets. These were each analysed as normal; thereafter, standard multiple imputation procedures were used to combine the multiple scalar and multivariate estimate quantities. There were no missing data in respect of the primary end-points of the study.

\section{Ethical consideration}

The present study, conducted according to the guidelines laid down in the Declaration of Helsinki, received ethical approval from the National Research Ethics Committee (reference no. 10/H0605/19) and approval from the local research and development department. Written informed consent was obtained from all patients before data collection and intervention.

The investigators comprised a research dietitian (S. W.), spinal cord physician (A. J.), microbiologist (J. O. D.), gastroenterologists (M. W., R. S. and A. F.), geriatrician (C. Y. Y.), nutrition scientist (G. G.) and health psychology statistician (S. P. H.). To monitor the progress and conduct of the study, all investigators attended meetings before the study, and met for communal annual updates in October 2011 (interim analysis) and October 2012 (end of the study meeting).

\section{Results}

The patient flow is summarised in Fig. 1. Over the 24 months of the study period, 167 SCI patients were approached by the principal investigator; three patients refused to participate in the study. Of the 164 patients who met the inclusion criteria and agreed to participate in the study, six patients withdrew during the study. Finally, 158 patients completed the study (mean age 50.1 (SD 19.1) years; 20.1\% female; $62 \cdot 5 \%$ tetraplegia; $37 \cdot 8 \%$ complete SCI; Table 1 ).

SCI was caused by traumatic injury (74.4\%) (fall: $n$ 59, 36.6\%; road traffic accident: $n$ 35, 21.7\%; sports-related injury: $n$ 23, 14.3\%; assault: $n 3,1.9 \%)$ and non-traumatic causes ( $n$ 41, 25.5\%).

The prevalence of risk for undernutrition was $65 \%$ ( $n$ 104) at the time of recruitment. Only one (25\%) in four was found to be missing one or more of their hospital meals. There were no difference observed between the LcS and control groups with regard to the daily intake of energy $(5774$ (SD 480.7) $v$. $5439(\mathrm{SD} 785.8) \mathrm{kJ}$ ), protein $(42.3(\mathrm{SD} \mathrm{3.8}) v .39 \cdot 5(\mathrm{sD} 5.4) \mathrm{g})$ and dietary fibre $(12 \cdot 4(\mathrm{SD} 3 \cdot 2) v .11 \cdot 6(\mathrm{SD} 2 \cdot 8) \mathrm{g}$ ).

Thirty-one (20.4\%) of 152 patients were prescribed PPI. Patients at risk of undernutrition were found to receive more prescribed medications than the low-risk group (13 v. 11, $P<0 \cdot 01$ ).

Most patients received one antibiotic (52.7\%), but $38.9 \%$ received two and $8.4 \%$ received three. A total of twentyeight different types of antibiotics were recorded in the present study: the oral route was used in $57.8 \%$ of patients and the intravenous route was used in $42.2 \%$ of patients. The median days of antibiotic were $21 \mathrm{~d}$ (range 5-366); no statistically significant differences with regard to antibiotic intake were observed between the LCS and control groups.

The reasons for starting the antibiotic treatment, in descending order, were as follows: urinary tract infection ( $n 74$, $45.1 \%)$; respiratory tract infection ( $n$ 46, 28.0\%); postoperative infection ( $n$ 19, 11.6\%); sepsis ( $n$ 5, 3.1\%); spinal tuberculosis treatment ( $n$ 4, 2.5\%); spinal abscess ( $n$ 4, $2.5 \%$ ); osteomyelitis ( $n 3,1.8 \%)$; Helicobacter pylori infection ( $n$ 2, 1.2\%); skin infection ( $n 2,1.2 \%$ ); other ( $n 5,3 \cdot 1 \%$ ).

At baseline, the LCS and control groups were similar with respect to demographic and clinical characteristics, which included: onset of SCI; proportions of tetraplegia and complete SCI; indications for antibiotic use; planned antibiotics (number and route); laxative use; PPI use; BMI; appetite and use of enteral feeding tubes (Table 2).

Patients in the control group received significantly more 'low-risk' antibiotics $\left(23.1\right.$ v. $\left.6.6 \%, \chi^{2}=8.429, P=0.004\right)$ and fewer 'high-risk' antibiotics (47.6 v. 63.2\%, $\chi^{2}=3.878$, $P=0.049)$ than those in the LcS group. No adverse events were reported in the present study. 
$\mathrm{SCl}$ patients newly started antibiotics, assessed for eligibility by the researcher n 167

Fig. 1. Study flow chart. SCI, spinal cord injury; LcS, Lactobacillus casei Shirota; od, once daily.

\section{Antibiotic-associated diarrhoea}

Overall, the incidence of AAD was $36 \%$. The incidence of AAD was significantly lower in the LcS group than in the control group $\left(17 \cdot 1\right.$ v. $\left.54.9 \% ; \chi^{2}=22 \cdot 39, P<0 \cdot 001\right)$.

\section{Clostridium difficile-associated diarrhoea}

Only one AAD case was associated with $C$. difficile infection (glutamate dehydrogenase positive; $C$. difficile toxin positive); this patient was in the control group.

Table 1. American Spinal Injury Association (ASIA) impairment classification of the participants*

\begin{tabular}{|c|c|c|c|c|c|}
\hline & \multicolumn{5}{|c|}{ Level of spinal cord injury } \\
\hline & \multirow[b]{2}{*}{ Cervical $(n)$} & \multirow[b]{2}{*}{ Thoracic $(n)$} & \multirow[b]{2}{*}{ Lumbar $(n)$} & \multicolumn{2}{|c|}{ Total } \\
\hline & & & & $n$ & $\%$ \\
\hline \multicolumn{6}{|l|}{ AIS } \\
\hline$A$ & 31 & 29 & 1 & 61 & $37 \cdot 8$ \\
\hline B & 22 & 6 & 0 & 28 & 17.4 \\
\hline C & 31 & 12 & 6 & 49 & $30 \cdot 4$ \\
\hline D & 17 & 5 & 1 & 23 & 14.4 \\
\hline \multicolumn{6}{|l|}{ Total } \\
\hline$n$ & 101 & 52 & 8 & \multicolumn{2}{|c|}{161} \\
\hline$\%$ & $62 \cdot 7$ & $32 \cdot 3$ & 5.0 & & \\
\hline
\end{tabular}

AIS, American Spinal Injury Association Impairment Scale; $62.5 \%$, tetraplegia; $37.3 \%$, paraplegia; $37.8 \%$, complete spinal cord injury; $62.2 \%$, incomplete spinal cord injury.

* Three missing neurology and AIS due to the lack of routine measurement of readmitted patients.
Risk factors for antibiotic-associated diarrhoea/Clostridium difficile-associated diarrhoea

Patients at undernutrition risk who received routine care were found to have a significantly higher incidence of AAD than those at low risk of undernutrition who received routine care $\left(64 \cdot 1 v \cdot 33 \cdot 3 \%, \chi^{2}=7 \cdot 101, P=0 \cdot 008\right)$. We observed a lower incidence of AAD in the 'at-risk' patients in the LCS group than in those in the routine-care control group (15.7 v. 64.1\%, $\left.\chi^{2}=25.356, P<0.001\right)$.

In the LcS group, patients who took regular PPI were found to have a significantly higher incidence of AAD than those in the non-PPI group (38.4 v. $\left.12.1 \%, \chi^{2}=5.627, P=0.022\right)$ There were no statistically significance differences observed in patients in the control group $\left(56 \cdot 3 v \cdot 50 \cdot 9 \%, \chi^{2}=0 \cdot 142\right.$, $P=0 \cdot 707)$.

The univariate linear regression analysis revealed that the number of drugs (OR 1.19, 95\% CI 1.06, 1.32), the number of antibiotics (OR 1.96, 95\% CI 1.12, 3.42), nutrition risk score (OR 1·15, 95\% CI 1.05, 1.26), serum albumin concentration (OR 0.90, 95\% CI 0.84, 0.96), mechanical ventilation (OR 0.30, 95\% CI 0.12, 0.77), gastrostomy feeding (OR 0.10, 95\% CI 0.01, 0.91), nil-by-mouth status (OR 0.18, $95 \%$ CI $0.068,0.46)$, poor appetite $(<1 / 2)$ (OR 4.96, $95 \%$ CI 1.65 , 14.91 ) and being in the control (not LCS) group (OR 5.45, $95 \%$ CI 2.62, 11.35) were all associated with increased AAD. The binary multivariate logistic regression analysis identified reduced appetite $(<1 / 2)$ (OR 5.04, 95\% CI 1.28, 19.84) and being in the control group (OR $8 \cdot 46,95 \%$ CI 3.22, 22.20) as the unique risk factors for AAD (Table 3 ). 
Table 2. Baseline characteristics of the study participants

(Number of patients and percentages or median values)

\begin{tabular}{|c|c|c|c|c|c|}
\hline & \multicolumn{2}{|c|}{$\begin{array}{l}\text { LcS group } \\
\quad(n 76)\end{array}$} & \multicolumn{2}{|c|}{$\begin{array}{l}\text { Control } \\
\text { group } \\
(n 82)\end{array}$} & \multirow[b]{2}{*}{$P$} \\
\hline & $n$ & $\%$ & $n$ & $\%$ & \\
\hline \multicolumn{6}{|l|}{ Parameters } \\
\hline Age (years) & \multicolumn{2}{|c|}{52.5} & \multicolumn{2}{|c|}{51} & 0.657 \\
\hline Male & 62 & 81.6 & 69 & $84 \cdot 1$ & 0.948 \\
\hline Caucasian & 67 & $88 \cdot 1$ & 72 & $84 \cdot 7$ & 0.524 \\
\hline \multicolumn{6}{|c|}{ Primary diagnosis on recruitment } \\
\hline Onset of $\mathrm{SCl}$ (days) & \multicolumn{2}{|c|}{71} & \multicolumn{2}{|c|}{60} & 0.474 \\
\hline Tetraplegia & 48 & 63.2 & 52 & 63.4 & 0.631 \\
\hline Complete SCl & 27 & 35.5 & 35 & $42 \cdot 7$ & 0.502 \\
\hline Median number of laxatives & \multicolumn{2}{|c|}{2} & \multicolumn{2}{|c|}{2} & 0.735 \\
\hline Median number of drugs & \multirow{2}{*}{\multicolumn{2}{|c|}{12}} & \multirow{2}{*}{\multicolumn{2}{|c|}{$\begin{array}{c}12 \\
1\end{array}$}} & 0.537 \\
\hline Median number of antibiotics & 1 & & & & 0.949 \\
\hline Antibiotic route: oral & 26 & 34.2 & 24 & $29 \cdot 3$ & 0.126 \\
\hline \multicolumn{6}{|l|}{$\begin{array}{l}\text { Risk of antibiotics causing } \\
\text { diarrhoea* }\end{array}$} \\
\hline Low & 5 & $6 \cdot 6$ & 19 & $23 \cdot 1$ & 0.004 \\
\hline Medium & 25 & 32.9 & 24 & $29 \cdot 3$ & 0.622 \\
\hline High & 48 & 63.2 & 39 & $47 \cdot 6$ & 0.049 \\
\hline \multicolumn{6}{|l|}{ Indication of antibiotics } \\
\hline Urinary tract infection & 32 & $42 \cdot 1$ & 42 & $50 \cdot 0$ & 0.320 \\
\hline Respiratory tract infection & 20 & $26 \cdot 3$ & 26 & $31 \cdot 7$ & 0.456 \\
\hline Use of PPI† & 14 & $18 \cdot 9$ & 17 & $21 \cdot 8$ & 0.532 \\
\hline Risk of undernutrition $\ddagger$ & 51 & 68 & 53 & 62.4 & 0.759 \\
\hline \multicolumn{6}{|l|}{ Nutrient intake } \\
\hline Energy (kJ) & \multicolumn{2}{|c|}{$5777 \cdot 7$} & \multicolumn{2}{|c|}{$5442 \cdot 8$} & 0.536 \\
\hline Dietary fibre (g) & \multicolumn{2}{|c|}{$12 \cdot 4$} & \multicolumn{2}{|c|}{11.6} & 0.606 \\
\hline BMI $\left(\mathrm{kg} / \mathrm{m}^{2}\right)$ & \multicolumn{2}{|c|}{$24 \cdot 2$} & \multicolumn{2}{|c|}{23.7} & 0.589 \\
\hline Use of enteral feeding tube & 9 & 11.8 & 13 & $15 \cdot 2$ & 0.589 \\
\hline \multicolumn{6}{|c|}{$\begin{array}{l}\text { LCS, Lactobacillus casei Shirota; SCI, spinal cord injury; PPI, proton pump inhibitor. } \\
\text { *Low risk: metronidazole and parenteral aminoglycosides; medium risk: tetra- } \\
\text { cyclines, sulphonamides, macrolides and quinolones; high risk: aminopenicillin } \\
\text { and cephalosporins (Kelly \& LaMont }{ }^{(19)} \text { ). } \\
\text { tPPI: twelve missing data. }\end{array}$} \\
\hline
\end{tabular}

As only a small number of patients developed CDAD during the follow-up period, this form of analysis was deemed inappropriate for the CDAD data.

\section{Discussion}

This is the first randomised controlled trial with an adequate sample size, which has evaluated the efficacy of LcS for the prevention of AAD in patients with SCI. The number of AAD cases was significantly lower in the LcS group.

The present study found a higher incidence of AAD (36.1\%) than some previous reports (approximately $25 \%)^{(1)}$. This may be attributed to a longer follow-up period ( $30 \mathrm{~d}$ ) than in many of the other published trials (often only $7-14 \mathrm{~d}$ ) ${ }^{(22)}$ as diarrhoea may occur up to 2 months after discontinuing antibiotic treatment ${ }^{(4)}$.

The present study defined AAD as more than two liquid stools (Bristol Stool Chart type 5, 6 or 7) a day for more than $3 \mathrm{~d}$, in quantities in excess of normal. This was chosen on clinical grounds according to the recommendation of the Hospital Microbiology Department at the time of the study. This relatively stringent definition enabled us to differentiate between clinically relevant and clinically unimportant changes in the consistency of stools. Definitions of AAD, however, vary between published studies. For example, Hickson et al. ${ }^{(21)}$ defined diarrhoea as $\geq 2$ stools per $\mathrm{d}$ for $\geq 3 \mathrm{~d}$, whereas Stein et $a{ }^{(23)}$ defined diarrhoea as $\geq 2$ watery stools within $24 \mathrm{~h}$. The present results are nonetheless consistent with those of other trials performed on hospitalised adult patients ${ }^{(21,23)}$.

Current evidence indicates that some probiotic strains reduce the incidence of $\mathrm{AAD} / \mathrm{CDAD}$ in general hospitalised patients ${ }^{(21)}$; however, the effects are strain-, product-, doseand disease-specific ${ }^{(12-14,24)}$. It has been suggested that the dose of probiotics should exceed $10^{10} \mathrm{CFU} / \mathrm{d}$ in order to prevent $\mathrm{AAD}^{(12)}$. The present study dose of a minimum of $6.5 \times 10^{9}$ CFU LCS was selected on pragmatic grounds and to aid compliance, and it is possible that a higher dose might have yielded even greater benefit. However, the effect of increasing dosage of probiotics should be monitored carefully as unexpected adverse events may also occur.

An important criterion for any probiotic is that the strains used survive the passage through the stomach and arrive in a viable state in the small intestine and colon. LcS has been shown to survive and be well tolerated in the upper gastrointestinal tract and which reach the large intestine in a viable state, and several human intervention trials have shown the presence of LcS in stool samples following daily consumption of a commercially available probiotic yogurt drink ${ }^{(25-28)}$.

LCS is considered safe to be used in clinical settings and has been used in a broad range of patients ${ }^{(29-31)}$. Evidence from

Table 3. Multivariate logistic regression analysis to identify risk factors for antibioticassociated diarrhoea

(Standard errors, odds ratios and $95 \%$ confidence intervals)

\begin{tabular}{lcccc}
\hline & SE & OR & $95 \% \mathrm{Cl}$ & $P$ \\
\hline Number of drugs & 0.071 & 1.105 & $0.962,1.271$ & 0.158 \\
Number of antibiotics & 0.373 & 1.751 & $0.843,3.639$ & 0.133 \\
SNST score & 0.080 & 1.127 & $0.963,1.318$ & 0.137 \\
Albumin & 0.049 & 0.927 & $0.842,1.020$ & 0.119 \\
Mechanical ventilation & 0.745 & 0.594 & $0.138,2.557$ & 0.484 \\
Gastrostomy feeding & 1.415 & 0.468 & $0.029,7.490$ & 0.591 \\
Nil by mouth & 1.460 & 0.159 & $0.009,2.787$ & 0.208 \\
Poor appetite (<1/2) & 0.699 & 5.045 & $1.283,19.84$ & 0.021 \\
Probiotic control (routine care) & 0.492 & 8.459 & $3.224,22.196$ & $<0.0001$ \\
\hline
\end{tabular}

SNST, Spinal Nutrition Screening Tool. 
human studies suggests that LcS predictably increases the number of beneficial intestinal bacteria ${ }^{(28)}$. LcS has been shown to help with constipation ${ }^{(32)}$, modulate immune function $^{(33,34)}$, reduce global infection risk $^{(35)}$, inhibit H. pylori ${ }^{(36)}$ and reduce the risk of diarrhoea ${ }^{(37)}$.

Several probiotics have been used safely in patients ${ }^{(38)}$. The safety of LCS has been confirmed in a wide range of patients and disease states ${ }^{(29,31)}$, including critically ill children admitted for intensive care ${ }^{(30)}$.

The present study suggests that poor appetite, which can lead to undernutrition, is an independent risk factor for AAD. A significant number of patients tend to miss one or more of their hospital meals, and this can contribute to a substantial loss of energy. In the present study, meals were not consumed by one-third of the patients, indicating an important missed opportunity in SCI patients. Previous research has suggested that consumption of oral nutritional supplement by patients with known poor appetite could improve their clinical outcome ${ }^{(39)}$. Clinicians should be aware of the need to identify and treat undernutrition and thus prevent undernutrition-related complications.

Probiotic administration and intake of dietary fibre can influence the composition of the gut microbiota, and thus promotes the availability of SCFA in the colon. This could have a beneficial effect on reducing the onset of diarrhoea because SCFA are potent stimulators of colonic water and electrolyte absorption ${ }^{(15,40)}$. In the present study, nutrient intake was estimated by a food record chart, so it was not possible to perform individual dietary analyses. After taking an average of our hospital menu, however, we found no statistical difference in the intake of energy, protein and dietary fibre between the two groups (LcS and control).

There are some limitations associated with the unblinded nature of the study. Due to the lack of a true placebo for the trial, use of a heat-treated LcS was considered while planning the present study, but the production and quality-control requirements for this approach were not possible. Heat treatment may not have killed all of the bacteria and may have led to undesirable taste changes in the material which would have affected compliance in the control group. Furthermore, heattreated bacteria can have some immune-modulatory effects. Therefore, we had little choice but to elect to have an openlabel study and use no intervention for the control group.

It should be noted that data for all of the bowel results were collected by trained nurses using validated criteria ${ }^{(17)}$ as part of their daily routine clinical assessment. Data on the occurrence of diarrhoea were subsequently collected by research staff according to the definition of diarrhoea. The nursing management was aware of but had no role in data collection; thus, we believe the present findings can be generalised to SCI patients receiving antibiotics.

In the present study, the encouragingly low incidence of CDAD probably reflects the adoption of the protocol recommended by the UK Department of Health that focuses on strict hygienic practices, strict antibiotic use, restricted use of laxatives and PPI, and isolation wards for confirmed and suspected $C$. difficile cases ${ }^{(5)}$. The numerical data do not contradict a possible additional benefit from LcS.
In conclusion, based on the present observation of a significant decrease in the incidence of AAD in patients with SCI, we can now consider LCS as a valid measure for the prevention of AAD in patients with SCI admitted to SCI centres. To confirm the present findings and evaluate the effectiveness of $\mathrm{LCS}$ in reducing the consequences of $\mathrm{AAD} / \mathrm{CDAD}$, a larger placebo-controlled study including different geographical locations is needed.

\section{Acknowledgements}

S. W. was supported by the Healthcare Infection Society (Mike Emmerson Young Investigator Award). Yakult UK Limited provided financial support for the trial. Yakult UK Limited provided free LCS, the necessary equipment to maintain the cold chain and a modest unrestricted educational grant. The company was aware of, but did not influence the trial design, and had no role in the data analysis and interpretation.

UCL Staff received support from the BRC funding awarded to UCL and its partner Trust by NIHR.

The authors would like to thank the patients and staff in the National Spinal Injuries Centre at Stoke Mandeville Hospital, especially Paul Subong, Lee Conway, Vanessa Stratfull, Maureen Coggrave, Debbie Green, Linda Dentice, Joost van Middendorp, Lisa Andrews, Anitha Niadoo, Breda Connolly and Pauline Bateman. We are also grateful to the medical consultants of the National Spinal Injuries Centre, Maurizio Belci, Fadel Derry, Allison Graham and Mofid Saif, for allowing us to recruit patients under their care for the study. We also thank Steven Cameron and Veronica Moore for proofreading the manuscript.

The authors' contributions are as follows: S. W. contributed to protocol development, data collection, data analysis and manuscript preparation; A. J., M. W., R. S. and C. Y. Y. contributed to protocol development, clinical supervision and manuscript revision; J. O. D. contributed to protocol development, clinical supervision, validation of laboratory data and manuscript revision, and acted as the guardian of laboratory data; S. P. H. contributed to protocol development, statistical supervision and manuscript revision; G. G. contributed to academic supervision and manuscript revision; A. F. contributed to academic supervision, data analysis and manuscript revision, and acted as the guarantor of the paper. Some of the study data were presented at the Healthcare Infection Society conference in Liverpool in November 2012 and at the American Spinal Injury Association annual conference in Chicago in May 2013.

\section{References}

1. Bartlett JG (2002) Clinical practice, antibiotic-associated diarrhoea. $N$ Eng J Med 346, 334-349.

2. Ackermann G, Thomalla S, Ackermann F, et al. (2005) Prevalence and characteristics of bacteria and host factors in an outbreak situation of antibiotic-associated diarrhoea. J Med Microbiol 54, 149-153.

3. Bergone-Berezin E (2000) Treatment and prevention of antibiotic-associated diarrhoea. Int J Antimicrob Agents 16 521-526

4. McFarland LV (2000) Normal flora: diversity and functions. Microb Ecol Health Dis 12, 193-207. 
5. Health Protection Agency/Department of Health (2008) Clostridium difficile Infection: How to Deal with the Problem. London: Department of Health.

6. Knoop FC, Owens M \& Crocker IC (1993) Clostridium difficile: clinical disease and diagnosis. Clin Micro Rev 6 , $251-265$

7. Katikireddi V (2005) UK launches inquired into Clostridium difficile outbreaks. CMAJ 173, 138.

8. Joint Food and Agriculture Organization of the United Nations (FAO) and the World Health Organization (WHO) (2002) Report of a Joint FAO/WHO Working Group on Drafting Guidelines for the Evaluation of Probiotics in Food. London, Ontario, Canada: FAO/WHO (April 30 and May 1).

9. Qamar A, Aboudola S, Warny M, et al. (2001) Saccharomyces boulardii stimulates intestinal immunoglobulin A immune responses to Clostridium difficile toxin A in mice. Infect Immun 69, 2762-2765.

10. Elmer GW (2001) Probiotics: "Living drugs". Am J Health Syst Pharm 58, 1101-1109.

11. Gibson GR, McCartney AL \& Rastall RA (2005) Prebiotics and resistance to gastrointestinal infections. Br J Nutr 93, Suppl. $1, \mathrm{~S} 31-\mathrm{S} 34$.

12. McFarland LV (2006) Meta-analysis of probiotics for the prevention of antibiotic associated diarrhoea and the treatment of Clostridium difficile diseases. Am J Gastroenterol 101, 812-822.

13. Wong SS, O'Driscoll J, Weldon M, et al. (2010) Nutritional status predicts hospital length of stay and mortality in patients with Clostridium difficile (C. diff) infection. Proc Nutr Soc 69 (OCE2), E175.

14. Hempel S, Newberry SJ, Maher AR, et al. (2012) Probiotics for the prevention and treatment of antibiotic-associated diarrhea. A systematic review and meta-analysis. JAMA 307, 1959-1969.

15. Pirker A, Stockenhuber A, Remely M, et al. (2012) Effects of antibiotic therapy on the gastrointestinal microbiota and the influence of Lactobacillus casei. Food Agricult Immunol (epublication ahead of print version 29 May 2012).

16. Consortium for Spinal Cord Medicine (2008) Early Acute Management in Adults with Spinal Cord Injury: A Clinical Practice Guideline for Health-care Providers. Washington DC: Paralyzed Veterans of America.

17. O'Donnell LD, Virjee J \& Heaton KW (1990) Detection of pseudo diarrhoea by simple clinical assessment of intestinal transit time. Br Med J 300, 439-440.

18. Hiesmaryr M, Schindler K, Pernicka E, et al. (2009) Decreased food intake is a risk factor for mortality in hospitalised patients: The NutritionDay Survey 2006. Clin Nutr 28, 484-491.

19. Kelly CP \& LaMont JT (1998) Clostridium difficile infection. Annu Rev Med 49, 375-390.

20. Wong S, Derry F, Jamous A, et al. (2012) Validation of the Spinal Nutrition Screening Tool (SNST) in patients with spinal cord injuries (SCI) - result from a multicentre study. Eur J Clin Nutr 66, 382-387.

21. Hickson M, D'Souza Al, Muthu N, et al. (2007) Use of probiotic Lactobacillus preparations to prevent diarrhoea associated with antibiotics: randomized double blind placebo controlled trial. BMJ 335, 80

22. Hickson M (2011) Probiotics in the prevention of antibioticassociated diarrhoea and Clostridium difficile infection. Ther Adv Gastroenterol 4, 185-197.

23. Stein GY, Nanim R, Kamiel E, et al. (2007) Probiotics as prophylactic agents against antibiotic-associated diarrhea in hospitalized patients. Harefuab 146, 520-522.

24. Sazawal S, Hiremath G, Dhingra U, et al. (2006) Efficacy of probiotics in prevention of acute diarrhoea: a meta-analysis of masked, randomised, placebo-controlled trials. Lancet Infect Dis 6, 374-382.

25. Yuki N, Watanabe K, Mike A, et al. (1999) Survival of a probiotic, Lactobacillus casei strain Shirota, in the gastrointestinal tract: selective isolation from faeces and identification using monoclonal antibodies. Int J Food Microbiol 48, $51-57$.

26. Lewis JN, Greig M, Candy DA, et al. (2002) Development of an ELISA to detect Lactobacillus casei Shirota in human stool samples. Br J Nutr 88, Suppl. 1, S113-S114.

27. Tuohy KM, Pinart-Gilberga M, Jones M, et al. (2007) Survivability of a probiotic Lactobacillus case $i$ in the gastrointestinal tract of healthy human volunteers and its impact on the faecal microflora. J Appl Microbiol 102, 1026-1032.

28. Matsumoto K, Takada T, Shimizu K, et al. (2010) Effects of a probiotic fermented milk beverage containing Lactobacillus casei strain Shirota on defecation frequency, intestinal microbiota, and the intestinal environment of healthy individuals with soft stools. J Biosci Bioeng 110, 547-552.

29. Matsuzaki T, Saito M, Usuku K, et al. (2005) A prospective uncontrolled trial of fermented milk drink containing viable Lactobacillus casei strain Shirota in the treatment of HTLV-1 associated myelopathy/tropical spastic paraparesis. J Neurol Sci 237, 75-81.

30. Srinivasan R, Meyer R, Padmanabhan R, et al. (2006) Clinical safety of a Lactobacillus casei Shirota as a probiotic in critically ill children. J Pediatr Gastroenterol Nutr 42, 171-173.

31. Stadlbauer V, Mookerjee RP, Hodges SJ, et al. (2008) Effect of probiotic treatment on deranged neutrophil function and cytokine responses in patients with compensated alcoholic cirrhosis. J Hepatol 48, 945-951.

32. Sakai T, Makino H, Ishikawa E, et al. (2011) Fermented milk containing Lactobacillus casei strain Shirota reduces incidence of hard or lumpy stools in health population. Int J Food Sci Nutr 62, 423-430.

33. Morimoto K, Takeshita T, Nanno M, et al. (2005) Modulation of natural killer cell activity by supplementation of fermented milk containing Lactobacillus casei in habitual smokers. Prev Med 40, 589-594.

34. Dong H, Rowland I, Thomas L, et al. (2013) Immunomodulatory effects of a probiotic drink containing Lactobacillus casei Shirota in healthy older volunteers. Eur J Nutr (epublication ahead of print version 10 January 2013).

35. Gleeson M, Bishop NC, Oliveira M, et al. (2011) Daily probiotic's (Lactobacillus casei Shirota) reduction of infection incidence in athletes. Int J Sport Nutr Exerc Metab 21, 55-64.

36. Sgouras D, Maraqkoudakis $\mathrm{P}$, Petraki K, et al. (2004) In vitro and in vivo inhibition of Helicobacter pylori by Lactobacillus casei strain Shirota. Appl Environ Microbiol 70, 518-526.

37. Sur D, Manna B, Niyogi SK, et al. (2011) Role of probiotic in preventing acute diarrhoea in children: a community-based, randomized, double-blind placebo-controlled field trial in an urban slum. Epidemiol Infect 139, 919-926.

38. Whelan K \& Myers CE (2010) Safety of probiotics in patients receiving nutritional support: a systematic review of case reports, randomized controlled trials, and nonrandomized trials. Am J Clin Nutr 91, 687-703.

39. Wong S, O'Driscoll J, Weldon W, et al. (2010) Do regular oral nutritional supplements improve clinical outcomes in patients with Clostridium difficile infection: a pilot study. Proc Nutr Soc 69 (OCE2), E174.

40. Ramakrishna BS, Venkataraman S, Srinivasan P, et al. (2000) Amylase resistant starch plus oral rehydration solution for cholera. N Eng J Med 342, 308-313. 